\title{
A Study on Cloud Computing: A Review
}

\author{
Swati Maurya ${ }^{1}$, Tabish Mufti ${ }^{2}$, Deepak Kumar ${ }^{3}$, Pooja Mittal $^{4}$, Rupam ${ }^{5}$ \\ \{maurya2026@gmail.com\} \\ 1,4,5 Department of Computer, Science and Engineering, SEST, Jamia Hamdard, New Delhi \\ ${ }^{2}$ Department of Computer Application, Mewar University, Chittorgarh, Rajisthan \\ ${ }^{3}$ Amity Institute of Information Technology, Amity University, Noida Uttar Pradesh
}

\begin{abstract}
In the field of technology, cloud computing becomes one of the fastest and growing technology. In cloud computing, users enable to access their data and resources on another machine rather than local servers via the internet and also allows to create an application and host them. Cloud providers are Amazon web services(AWS), Microsoft azure and Google cloud platform(GCP). Vendors provide services to end-users and the services are compute, storage, networking, analytics, etc. The main objective of this paper is to compare the different cloud providers on the basis of some parameters which are pricing, storage, availability zone and compute.
\end{abstract}

Keywords: Amazon Web service, Google cloud Platform, Microsoft Azure, Cloud Computing, Security

\section{Introduction}

The demand for cloud services have increased rapidly in recent years result in major boom in cloud platform user's scalability[1].Nowadays, everybody wants to keep their data on servers or sometimes they want to host some applications with agility and flexibility but how to do that. First of all, we need to purchase a server. After that Since we have so many servers so we have to maintain them. But the problem is that the setup is very expensive and also troubleshooting problems occur. To overcome this problem a technology called "cloud computing" has been established. Cloud computing [2][3] refers to a technology in which there is no need to purchase servers anymore simply users can access services that are provided by vendors.

List of top most cloud service providers:

- Google cloud platform

- Amazon web services

- Microsoft azure

Amazon web services were launched in 2006 when companies didn't have any cloud computing model in a market. AWS is a platform that is secure and serves many services like EC2 service, RDS, VPC, Route 53, Load Balancer. AWS becomes one of the best service providers due to its flexibility, scalability, security[4], and pricing. AWS is based on the pay-as-you-go model[5][6]. Users of Amazon web services are Netflix, Twitch, LinkedIn, Facebook, Adobe, etc.

Microsoft Azure is a cloud computing platform and was launched on 1 February 2010[7]. Microsoft azure is based on pay-per-use model which means users only need to pay only for the 
services they used. By using the services of Microsoft azure users can easily access their data and transform their data. Microsoft Azure has many datacenters across the world so that vendors can deliver their services throughout the world. Pixel, Apple iCloud, Xerox, Samsung, eBay are the customers of Microsoft azure[8][9].

Google cloud platform commences its journey on April 7, 2008. Google offers services such as storage, compute and networking. that runs on a platform that is infrastructure-as-a-service(Iaas) and platform-as-a-service(Paas)[10]. Google search and YouTube are Google products. Customers of a Google cloud platform: PayPal, Retail, HSBC, McKesson[11].

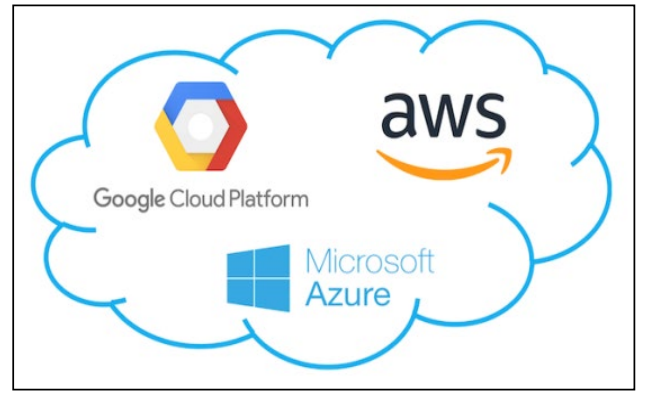

Figure 1. Cloud Service Providers

\section{Literature Review}

Pranay Dutta and Prashant Dutta(2019) proposed the study of Amazon web services(AWS)[12], Microsoft Azure and Google cloud platform(GCP) and give a deep dive into the comparison of AWS,MA and GCP on the basis of a database, management, networking and security services [13][14]. As stated by Synergy Research Group in the cloud infrastructure market that Amazon web services hold 33 percent, Microsoft Azure improved from 10 percent to 13 percent and Google Cloud Platform increased 5 to 6 percent. Amazon has a variety of services to offer but its pricing schemes are over expensive side where Microsoft azure is reliable but it lacks in the market in terms of development. Meanwhile, Google Cloud Platform has expertise in the development offers flexible discounts and specially designed for small scale businesses but it does not offer as many services as Amazon web service and Microsoft azure.

Arabolu Chandra Sekhar and Dr. R.Praveen Sam(2015)studied Amazon web service is the world's most adopted cloud platform and the first vendor to enter the cloud computing market which offers 165 services with storage, security, database, analytics, application and development services. Some of the services are cloud front, cloud formation, elastic compute cloud(EC2), dynamo DB, simple storage service, route 53, elastic cache, elastic beanstalk, etc. and the most commonly used services are simple storage service(s3) and Amazon EC2. Amazon web service is located in 8 regions Singapore, Sydney, Ireland, Brazil and Amazon are planning to add five more AWS 
region in Indonesia, Japan, Spain, Italy, and South Africa. Organizations are dealing with IT infrastructure in a new way due to Amazon's Elastic Compute and IBM's smart cloud services. Amazon web service was the first cloud provider so it has more experience as compared to other providers and schemes of Amazon web service are flexible, agility and scalability.

\section{Google cloud Architecture}

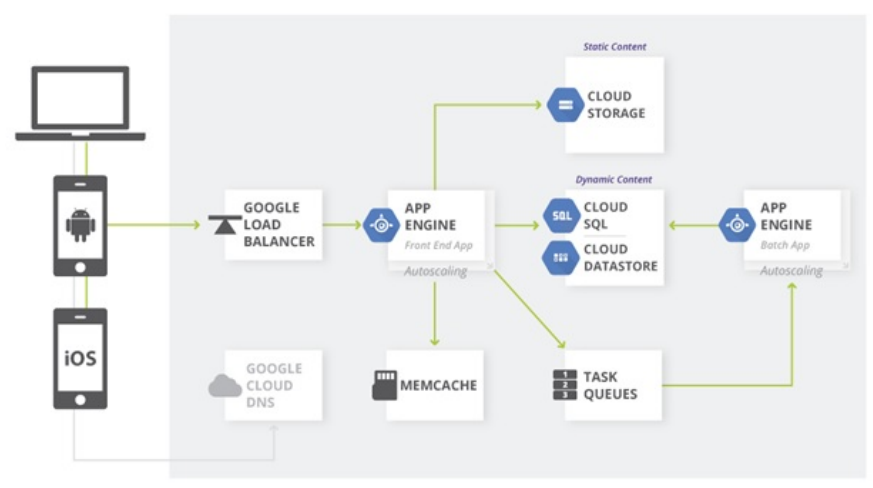

Figure 2. Google cloud Architecture platform (GCP)[15]

Cloud computing is not innovated as technology or architecture bit it is said to be information technology where delivery models are provided and each and every delivery model and specific type of architecture to compute different type of online resources

Services provided in such type of architecture are elastic in naute which means on the bsiss of services usablity the paymenrt is been made. A cloud can be of any type like public,private or hybrid.when the resources are shared publically then the cloud isp rivate when the resources are shared privately then the cloud is public rivate when the resources are shared publicallyprivatelythen the cloud is hybrid

\subsection{Google Load Balancer:}

Google Cloud Platform's Load Balancing simplifies the deployment [16] and seamlessly delivers the scale and high-availability according to our need. It can easily handle 150,000 requests per second [17][18] with no warm-up time and no preparation needed. Having this peace of mind has made a dramatic difference compared with days of configuring specialized load balancing hardware[19]. 


\subsection{Google Cloud DNS:}

Google Cloud DNS is an ascendable, reliable, and managed authoritative Domain Name System [20] (DNS) service running on a similar infrastructure as Google. It's low latency, high convenience and also cost-efficient helps to build the applications and services out there to the users.

\subsection{App Engine:}

Google App Engine is the Platform forthe Service and for cloud computing platform for the development and hosting the web applications in Google. Applications are the sand-boxed and to run across the multiple servers[21].

\subsection{Google Cloud Storage:}

It is the online File Storage service, which helps users to access their data from anywhere with regards to the internet connection. GCS provides scalability[22] and performance with advanced security and sharing capabilities.

\subsection{Google Task Queues:}

GTQ let applications to perform work known as tasks, unsynchronized of user requests. If any applications want to perform any task in the background then task adds task to task queues

\section{Features Cloud Computing}

1. On Demand Service

2. Compatibility

3. Portability

4. Elastic in Nature

5. Backup

6. Recovery

7. Economical

8. Easy Maintenance

9. Pay as you go

10. Security

\section{Comparison:}


Availability zone: The availability zone gives a capability for customers to build highly available applications in a region. Each availability zone consists of one or more datacenters and they are independent[23].

Storage: Storage is one of the main factors in cloud computing. To store and manage data is like a [24][25]big challenge, hosting websites online, delivery of content or distribution, etc. this takes a lot of space so what will be the better way?

Amazon provides a way to store data effectively and in a good manner by using "Simple Storage Service(S3)". Simple storage service deliver safe and secure object storage to the organization.

Sometimes some applications need shared file storage and that application can be accessed by many computers at the same time[26][27] but building own storage file system can be very expensive and consuming lots of time. So a better way to this using the "Amazon Elastic File System". Elastic File System manages all the infrastructure and automatically makes space for files as adding or removing files. In EFS, we only pay for the space our files use.

Microsoft Azure: Blob storage and data lake storage are the services that are given by Microsoft azure. Blob storage is a collection of binary data it can be anything like image, text, audio, etc. For big data applications, the Data Lake store has been used. In data lake store data can be stored in a native format. Data lake store is based on pay-as-per-use. SQL Database, Database for MySQL [28] and database for Postgre SQL are three SQL based options. Microsoft provides backup service and site recovery service and archive.

\section{Google storage:}

Google cloud platform has less number of storage services as compared to Microsoft azure and Amazon web services. From the database side, Google cloud platform has SQL based which is Cloud SQL. Cloud Spanner is a relational database that is used for mission-critical workloads. Having two NoSQL options: Cloud Bigtable and Cloud Datastore. google cloud platform does not support backup and archive services[29].

\section{Pricing:}

Amazon web services has a flexible pricing model. AWS takes charges only for the resources which we using. In AWS, we pick more services by paying less[30]. Microsoft azure consist of three types of subscription are free, pay-as-you-go and member offers. Pay-as-you-go describes that the user pays on a monthly basis for services and resources. Member offers like MSDN Platform subscribers, Visual Studio subscribers, etc are designed to reduced rates for services.

\section{Conclusion}

In today's hi-tech competitive world, at every seconds users are generating various types of data over the internet. Day by day and second by second this data is growing at rapid rate. At present scenario 2.5 quintillion bytes of data is generated in 24 hours of single day. So we need better tool to handle and manage data because storage of data will be major issue. So in this paper we have 
discussed about cloud technology where data is backed on virtual machine along with the architecture, advantages and comparison

\section{References}

[1] Hai Jia Jia Research on User Data Storage Security in Cloud Environment[D] Heilongjiang University 2015.

[2] Feng Chao-Sheng Qin Zhi-Guang Yuan Ding "Cloud data secure storage technology [J]" Journal of Computer Science no. 01 pp. 150-163 2015

[3] L. Logeswaran, H. M. N. D. Bandara, and H. S. Bhathiya, "Performance, Resource, and Cost Aware Resource Provisioning in the Cloud," in 2016 IEEE 9th International Conference on Cloud Computing (CLOUD), 2016, pp. 913-916.

[4] Q. Wang C. Wang J. Li K. Ren W. Lou "Enabling public verifiability and data dynamics forstorage security in cloud computing [C]" 14th European Symposium on Research in Computer Security pp. 355-370 2009.

[5] A. Ibrahimi and P. D. Student, "Cloud Computing: Pricing Model," vol. 8, no. 6, pp. 434$441,2017$.

[6] D. Chaudhary and B. Kumar, "Cost optimized Hybrid Genetic-Gravitational Search Algorithm for load scheduling in Cloud Computing," Appl. Soft Comput. J., vol. 83, 2019.

[7] Wenhong Tian Yong Zhao "An Introduction to Cloud Computing [J]" Cloud Resource Management and Scheduling pp. 1-15 2015.

[8] M. Klems, J. Nimis, and S. Tai, "Do clouds compute? A framework for estimating the value of cloud computing," vol. 22 LNBIP. Springer Verlag, Forschungszentrum Informatik (FZI), Haid-und-Neu-Str. 10-14, Karlsruhe 76131, Germany, pp. 110-123, 2009.

[9] C. Zhang, A. Yin, Y. Wu, Y. Chen, and X. Wang, "Fast Time Series Discords Detection with Privacy Preserving," 2018, pp. 1129-1139.

[10] T. C. Y. Chui, D. Siuta, G. West, H. Modzelewski, R. Schigas, and R. Stull, "On producing reliable and Affordable numerical weather forecasts on public cloud-computing infrastructure," J. Atmos. Ocean. Technol., vol. 36, no. 3, pp. 491-509, 2019.

[11] X. Yang, S. Zhu, and X. Pan, "Improved verifiability scheme for data storage in cloud computing," Wuhan Univ. J. Nat. Sci., vol. 16, no. 5, pp. 399-404, 2011.

[12] J. Ellman, N. Lee, and N. Jin, "Cloud computing deployment: a cost-modelling casestudy," Wireless Networks. Springer New York LLC, Department of Computer and Information Sciences, Faculty of Engineering and Environment, Northumbria University, Newcastle upon Tyne, United Kingdom, 2018.

[13] M. Sahinoglu, S. Ashokan, and P. Vasudev, "CLOUD computing: Cost-effective risk management with additional product deployment," 2015, vol. 62, pp. 319-325.

[14] B. Rochwerger et al., "The Reservoir model and architecture for open federated cloud computing,” IBM J. Res. Dev., vol. 53, no. 4, 2009.

[15] X. Liu, "Cloud architecture learning based on social architecture," 2011, pp. 418-421.

[16] A. Bouayad, A. Blilat, N. E. H. Mejhed, and M. El Ghazi, "Cloud computing: Security challenges," 2012, pp. 26-31. 
[17] S. Midya, A. Roy, K. Majumder, and S. Phadikar, "Multi-objective optimization technique for resource allocation and task scheduling in vehicular cloud architecture: A hybrid adaptive nature inspired approach," J. Netw. Comput. Appl., vol. 103, pp. 58-84, 2018.

[18] A. Behl and K. Behl, "An analysis of cloud computing security issues," 2012, pp. 109114.

[19]. Mehdi Bahrami Mukesh Singhal S.-M. Chen "The Role of Cloud Computing Architecture in Big Data" Information Granularity Big Data and Computational Intelligence vol. 8 pp. 275-295 2015.

[20]. Mehdi Bahrami Mukesh Singhal A Light-Weight Permutation based Method for Data Privacy in Mobile Cloud Computing in 2015 3rd Int. Conf.3rd IEEE International Conference on Mobile Cloud Computing Services and Engineering (IEEE Mobile Cloud 2015) San Francisco IEEE 2015.

[21] Mehdi Bahrami Mukesh Singhal Zixuan Zhuang "A Cloud-based Web Crawler Architecture" in 2015" 18th Int. Conf. Intelligence in Next Generation Networks: Innovations in Services Networks and Clouds (ICIN 2015) Paris France IEEE 2015.

[22] Mehdi Bahrami "Cloud Template a Big Data Solution" International Journal of Soft Computing and Software Engineering [JSCSE vol. 3 no. 2 pp. 13-17 2013

[23] https://phoenixnap.com/blog/orchestration-vs-automation.

[24] DIAO Zhe WANG Qinghong SU Naizheng ZHANG Yuhan "Study on Data Security Policy Based On Cloud Storage" 2017 IEEE 3rd International Conference on Big Data Security on Cloud..

[25] T. Subha Dr. S. Jayashri "Efficient Privacy Preserving Integrity Checking Model for Cloud Data Storage Security" 2016 IEEE Eighth International Conference on Advanced Computing (ICoAC).

[26] M. S. Binu J. Meenakumari "A security framework for an enterprise system on cloud" Indian Journal of Computer Science and Engineering (IJCSE) vol. 3 no. 4 pp. 548-552 2012.

[27] Mrinal Sarkar Sanjay Kumar "Ensuring data storage security in cloud computing based on hybrid encryption schemes" 2016 fourth international conference on parallel distributed \&amp; grid computing.

[28] Suresh Pabboju K Venkatesh Sharma "Data Security in Cloud computing and Outsourced Databases" Shankar Nayak Bhukya" International Conference on Electrical Electronics and Optimization Techniques (ICEEOT) 2016.

[29] Feng Deng-Guo Zhang Min Zhang Yan "Study on cloud computing security" Journal of Software vol. 22 no. 1 pp. 71-83 2011.

[30] Bai Xin "Research on Key Technology of Safety Monitoring Mechanism in Cloud Environment[D]" Beijing industry university 2015. 